\title{
COMUNICAÇÃO NO CONTEXTO DE REABILITAÇÃO: $O$ encontro entre enfermeiro e paciente
}

\author{
Communication at rehabilitation context: An encounter between nurse and patient
}

\author{
Beatriz Medeiros Martins ${ }^{a}$, Tereza Cristina Cavalcanti Ferreira de Araujo ${ }^{b}$
}

\footnotetext{
a Mestre em Psicologia pela Universidade de Brasília, Brasília, DF - Brasil, e-mail: beatriz.martins@tjdft.gov.br

b Professora da Universidade de Brasília. Pós-Doutora pela Unesco, Doutora pela Université de Paris X - Nanterre, Pesquisadora do Conselho Nacional de Desenvolvimento Científico e Tecnológico-CNPq, Brasília, DF - Brasil, e-mail: araujotc@unb.br
}

\begin{abstract}
Resumo
A qualidade da comunicação em saúde tem sido associada à satisfação com a prática assistencial por parte de pacientes e profissionais. Considerando tal perspectiva, a presente pesquisa teve por objetivo ampliar a compreensão sobre a comunicação enfermeiro-paciente no contexto de reabilitação. Para tanto, realizou-se registro das interações verbais em situação natural (procedimento de curativo) entre quatro díades paciente-enfermeiro. Em seguida, enfermeiros e pacientes foram entrevistados, individualmente, por meio de um roteiro com questões específicas. Posteriormente, foi desenvolvida uma entrevista focalizando questões gerais sobre o atendimento em reabilitação. Os resultados indicaram que os profissionais expressam conceitos e valores compatíveis com o modelo biopsicossocial de saúde, mas apresentam uma atuação com características do modelo biomédico, priorizando a informação em detrimento dos aspectos relacionais. Quanto aos pacientes, priorizam prestar as informações solicitadas e se comunicam utilizando recursos referenciados em seus valores e sentimentos (opiniões e expressão emocional). Para as díades enfermeiro-paciente, os aspectos relacionais e assistenciais são considerados mais relevantes do que a condição física no momento do atendimento. As variáveis "condição emocional do paciente" e "organização do trabalho" revelaram-se determinantes na disponibilidade do enfermeiro para a comunicação. Discutem-se questões associadas ao treinamento profissional e sugerem-se mais estudos sobre o tema, em especial aqueles que adotem técnicas observacionais com abordagem multimetodológica.
\end{abstract}

Palavras-chave: Comunicação em saúde; Reabilitação; Formação profissional. 


\begin{abstract}
The quality of the communication in health care has been associated to satisfaction of patients and professionals with assistancial practice. Considering this perspective, this research aims to extend the comprehension about the nurse-patient communication in the rehabilitation context. Verbal interactions in natural situation (banding procedure) among four pairs nurse-patient were registered, followed by interviews, aiming to analyze their perceptions about the therapeutic encounter. Each subject was interviewed about specific topics of that therapeutic intervention. Later, a second interview was developed, which focused on general aspects of the rehabilitation program. The results indicated that the professionals express concepts and values compatible to the biopsicosocial health model, but present an action with characteristics of the biomedical model, standing out the information to the detriment of relational aspects. Patients mainly give the information requested and communicate utilizing resources based on their own values and feelings (opinions and emotion expression). For the dyads, the relational and assistance aspects were considered more relevant than the physical conditions during their meeting. The variables "emotional state of the patient" and "work organization" showed as determinants in the nurse disponibility for communication. Questions related to professional training are discussed and it's indicated that new studies should be held, especially the ones that adopt observational techniques with a multimethodological approach.
\end{abstract}

Keywords: Health communication; Rehabilitation; Professional training.

\section{INTRODUÇÃO}

A comunicação é um importante mediador da qualidade da relação profissional de saúdepaciente. $\mathrm{Na}$ esfera dos cuidados em saúde, as interações se dão por meio de trocas, nas quais as produções verbais, as modulações vocais, as mímicas, os gestos, os olhares se combinam em partes variáveis e de acordo com regras associativas e seqüenciais, para constituir um enunciado total. A comunicação interpessoal serve para realizar outros atos de comunicação além do dizer e as interações são muito dependentes do contexto, que é constituído pelos participantes, a situação, a relação e a plataforma comunicativa comum entre eles (Cosnier, Grosjean \& Lacoste, 1994).

Compreender o processo de comunicação e desenvolver habilidades comunicacionais é tarefa necessária e fundamental a todo e qualquer agente que atue na área. Contudo, formas ambíguas de comunicação são utilizadas com freqüência nas instituições de saúde. Muitas das dificuldades estão relacionadas a "não saber ouvir" e usar linguagem inacessível ao receptor, como, por exemplo, imposições de ordens e lições de moral; ameaças e sugestões que não podem ser cumpridas; negação da percepção do outro; expressão de falso apoio; aferições de críticas generalizadas; elogios que soam como manipulação; fazer perguntas e não esperar respostas e emissão de respostas contraditórias entre o nível verbal e o não-verbal (Silva, 1996; Sliwa, Makoul \& Betts, 2002; Takman \& Severison, 1999; Tran, Haidet, Street Jr., O’Malley, Martin, \& Ashton. 2004; Waitzkin, 1990).

Em um estudo com médicos realizado ao longo de oito anos, identificaram-se modalidades patológicas de comunicação e relação. Os dados revelaram que tempo de atuação e treinamentos técnicos não modificam a conduta comunicacional. Não se verificou diferença de gênero. Em momentos diferentes, o mesmo médico e o mesmo paciente apresentaram padrão comunicacional similar. Foram freqüentes as distorções que comprometem a colaboração do paciente, interferindo no diagnóstico e provocando condutas terapêuticas equivocadas. Caracterizam as desqualificações: declarações contraditórias, mudanças bruscas de assunto, tangencializações, frases incompletas, interpretações errôneas, estilo obscuro, maneirismo de fala e interpretações metafóricas literais (Abdo, 1996).

Diversos estudiosos alertam que o reconhecimento do impacto da comunicação tem sido menor do que o devido. A literatura sobre o tema ainda é escassa e aquela já disponível é fragmentada. 
A ausência de um modelo integrado de comunicação no domínio de uma mesma disciplina científica explica em parte tal limitação. Os estudos são desenvolvidos em diferentes áreas do conhecimento e tendem a focalizar determinados elementos do processo de comunicação, em particular a díade médico-paciente. A ênfase no conteúdo, mais do que no contexto (como, por exemplo, nos aspectos institucionais) também preocupa os pesquisadores que defendem a elaboração de paradigmas para settings específicos. Eles consideram que diante da carência desses fundamentos, a atuação profissional acaba por ficar orientada por percepções pessoais de ordem prática, as quais costumam não serem suficientes para superar deficiências e insatisfações. Em outras palavras, a natureza da comunicação entre profissional e paciente ainda é um desafio na assistência em saúde (Grant, Cissna \& Rosenfeld, 2000; Inui \& Carter, 1985; Jones, 1999; Kaplan, 1997; Lamers \& Geist, 1997; Sharf \& Street Jr., 1997).

Mas uma análise pormenorizada da produção científica, permite verificar que muitas problemáticas centrais da área têm sido abordadas a partir do enfoque comunicacional, como, por exemplo, tomada de decisão, satisfação com atendimento e qualidade de serviços, adesão e enfrentamento. Quanto aos aspectos metodológicos, nota-se o predomínio de técnicas observacionais visando a construção de sistemas de análises interacionais a partir de registros em situação natural, complementados por entrevistas (Grant et al., 2000; Kaplan, 1997; Lambert et al., 1997; Lamers \& Geist, 1997; Makoul, Artson \& Shofield, 1995; Ramos, 1998; Sharf \& Street Jr., 1997; Takman \& Severinson, 1999). A situação de observação mais freqüente ainda é a consulta médica diádica (Cosnier et al., 1994; Grant et al., 2000; Makoul et al., 1995; McNeilis, 2001; Shaikh, Knobloch \& Stiles, 2001; Stewart \& Brown, 2001; Street Jr. \& Millay, 2001). O registro em vídeo, apesar de constituir-se como o recurso mais indicado para a observação da interação comunicacional, encontra dificuldades em ser efetivado e é empregado em alguns poucos estudos (Cosnier et al., 1994; Queiroz \& Araujo, 2007; Makoul et al., 1995).

Então, em convergência com este panorama de estudos sobre comunicação em saúde - indicador da relevância científica e profissional da temática - desenvolveu-se uma investigação visando analisar e compreender o encontro entre enfermeiro e paciente no contexto de reabilitação
(Kennedy, Marsh, Lawe, Grey, Short \& Rogers, 2000; Queiroz \& Araujo, 2007; Pereira \& Araujo, 2005; Reilly, 2001; Trexler \& Fordyce, 2000). Para tanto, estabeleceram-se como objetivos específicos: descrever os elementos comunicacionais e interacionais da díade enfermeiropaciente durante o procedimento de curativo. Identificar os fatores que facilitam e dificultam a interação em situação de cuidado. Comparar as percepções de cada membro da díade sobre o encontro terapêutico.

\section{MÉTODO}

\section{Caracterização dos participantes e do contexto}

Participaram da investigação três pacientes do sexo masculino e um do feminino, com idade variando entre 25 e 44 anos. Três possuíam diagnóstico de tetraplegia e um de paraplegia, em regime de internação para seguimento do programa de reabilitação. Entre os profissionais de saúde, participaram três enfermeiros, sendo dois do sexo feminino e um do sexo masculino, com tempo de trabalho na instituição variando de quatro meses a um ano. No total, foram estudadas quatro díades, envolvendo sete participantes.

O trabalho foi desenvolvido em uma unidade hospitalar da rede pública destinada a reabilitação de doentes crônicos, previamente diagnosticados e atendidos em outro hospital da rede. Portanto, apesar do paciente ter pouco tempo de internação, já possui experiência anterior de hospitalização. Compõem a equipe multiprofissional de assistência: médicos clínicos, fisiatras e hematologistas; enfermeiros e auxiliares; nutricionistas; assistentes sociais; psicólogos; fisioterapeutas; terapeutas ocupacionais e professoras. A equipe de enfermagem, foco da pesquisa, executa uma rotina de intervenção envolvendo, sobretudo, entrevista de admissão, curativos, cateterismo e orientações de autocuidado.

\section{Procedimentos para coleta de dados}

O projeto de pesquisa foi submetido a um Comitê de Ética reconhecido pelo Ministério da Saúde. Após a aprovação, o projeto foi entregue e discutido com a diretoria do hospital, que 
autorizou a coleta. Antes de iniciar esta etapa, foi realizado contato com a chefia de enfermagem, explicitando os objetivos gerais da pesquisa e os procedimentos da coleta. De modo similar, os participantes foram convidados e expressaram seu consentimento livre e esclarecido.

Elaborou-se um protocolo de observação contendo: a) dados gerais sobre o paciente (extraídos do prontuário); b) sessão de observação propriamente dita; e c) anotações complementares sobre eventos relevantes ocorridos durante a observação (por exemplo, comportamentos nãoverbais, interrupções, entrada e saída de pessoas da enfermaria). $O$ atendimento de curativo foi selecionado para sessão de registro por diversas razões: 1) é a modalidade assistencial com maior grau de estruturação; 2) constitui uma exclusividade técnica do enfermeiro; 3) o tempo de execução é maior quando comparado a outras intervenções; 4) foi indicado pelos profissionais como sendo o momento preferencial para interagir. A realização do curativo se dá no leito do paciente, após o banho, no período da manhã. De modo geral, a posição é de decúbito ventral, o que pode dificultar a interação por restringir o contato visual. Tem duração média de 15 a 30 minutos, mas pode requerer até 60 minutos.

Quanto às entrevistas, adotaram-se duas modalidades: a) a primeira, denominada "Questões Específicas sobre o Atendimento", foi realizada logo após a observação, com os dois membros da díade; e outra, denominada "Questões Gerais sobre o Atendimento", foi desenvolvida em um dia aleatório, de acordo com a disponibilidade dos sujeitos.

\section{Procedimento para análise dos dados}

Elaborou-se um sistema múltiplo de categorias de acordo com as três etapas da coleta de dados. O primeiro sistema foi gerado pela observação, ao passo que o segundo e o terceiro, respectivamente, permitem compreender a percepção de profissionais e pacientes a partir dos relatos de entrevista sobre o atendimento observado e acerca da experiência de reabilitação.

O sistema 1 foi criado a partir da elaboração de um conjunto preliminar de categorias que gerou uma folha de registro onde os dados transcritos da observação foram tabulados e permitiu o ajustamento do sistema final de categorias. Já os sistemas 2 e 3 foram gerados a partir da análise de conteúdo das entrevistas com elaboração de categorias agrupadas por critério semântico.

\section{Sistema 1}

Ele abrange três subsistemas gerais: finalidade da comunicação, recursos comunicacionais e fluxo da comunicação. Tais subsistemas compreendem três categorias principais, organizadas em diversas subcategorias: a) incentivo à participação: solicitar informação específica, solicitar atuação e solicitar auxílio; b) incentivo à interação: solicitar opinião, solicitar reflexão, solicitar relato de estado subjetivo, socialização, integração, comunicar expressão emocional, comunicar opiniões, comunicar expectativas, comunicar incertezas, comunicar reações; c) comunicar informações: informar fatos, comunicar procedimentos, comunicar informações específicas, comunicar problemas, comunicar condutas, comunicar evolução, afirmativa, confirmativa orientar, comunicar justificativa.

O segundo subsistema refere-se ao modo como a comunicação é realizada, ou seja, aos recursos utilizados pelos interlocutores para se comunicar. Prevê duas categorias principais: a) facilitadores: saudações, vocativo, apreciação, interativo, descritivo, ampliador, expressivo, comentar, clarificativo, conclusivo, reflexivo, confirmativo, reformulador, mantenedor e finalização; b) perturbadores: indagador, diretivo, restritivo, contraditório, tangencialização, não responder, interrupção.

Já o terceiro subsistema descreve o fluxo da comunicação, distinguindo seqüências interativas e interrupções, perfazendo duas categorias: a) caracterização das seqüências interativas: iniciativa da emissão e utilização dos perturbadores comunicacionais; b) caracterização das interrupções: pausa ou demanda externa.

\section{Sistema 2}

Permite a análise da percepção do enfermeiro e do seu paciente quanto ao procedimento de curativo. É composto por nove categorias (discriminadas em 81 subcategorias), focalizadas nesse atendimento específico, a saber: descrição do atendimento, objetivo, interferências, situações informativas e de orientação, adesão às orientações, percepção dos enfermeiros sobre os 
pacientes e estratégias relacionais adotadas, percepção dos pacientes em relação aos enfermeiros, satisfação com o atendimento e sugestões para melhorá-lo.

\section{Sistema 3}

Reúne as categorias relativas às percepções sobre o atendimento em geral, distinguindo-as de acordo com os membros da relação terapêutica: a) do enfermeiro: conceito de reabilitação, trabalho em equipe, especificidades da clientela e do tratamento, satisfação e frustração com o trabalho, papel da instituição na consecução da reabilitação; b) do paciente: assimilação da incapacidade e suas conseqüências, irreversibilidade do quadro, demanda de tratamento especializado, impacto da hospitalização, confiança no saber profissional, temporalidade do aprendizado, situações de orientação, estratégias de enfrentamento de problemas, avaliação da qualidade assistencial.

\section{RESULTADOS E DISCUSSÃO}

\section{1) Finalidade da comunicação e recursos comunicacionais}

A troca de informações é a finalidade predominante, contrapondo-se ao incentivo à interação. O profissional prioriza questões técnicas (procedimentos e problemas de funcionamento da equipe de enfermagem), em detrimento da orientação ao paciente, que por sua vez, limita-se a responder à solicitação de informação pelos enfermeiros. Vale salientar que também aparecem de forma significativa as comunicações sobre os problemas com a equipe de enfermagem. Quanto à categoria "incentivo à participação", a atuação do enfermeiro também prepondera sobre a do paciente. Solicitação de informações é o único recurso utilizado pelo paciente e aquele mais freqüentemente adotado pelo profissional.

$\mathrm{O}$ investimento em interagir durante o atendimento é maior por parte dos pacientes, que priorizam a comunicação de opiniões, de expressão emocional e reações como recursos para buscar a interação, enquanto os enfermeiros utilizam a socialização, solicitação de estado subjetivo e reflexão. Os enfermeiros apresentam-se com melhor utilização dos recursos comunicacionais do que os pacientes, tanto pela maior porcentagem de recursos promovedores de comunicação como pela menor utilização de recursos que dificultam. Entretanto, isso não significa que a utilização destes recursos pelos enfermeiros seja satisfatória. Uma análise detalhada indica que uma porcentagem maior de recursos é utilizada em relação a questões técnicas como indagar sobre informações específicas do tratamento. Em contrapartida, apesar de emitirem uma menor quantidade de promovedores comunicacionais, os pacientes empregam mais freqüentemente respostas que não têm a finalidade de responder a uma pergunta, mas de favorecer o contato e a permanência do diálogo (categoria "mantenedora").

\section{2) Fluxo da Comunicação}

Díade 1: a comunicação estabelecida entre esta díade apresenta uma performance melhor por parte do paciente do que do enfermeiro, pois aquele utiliza mais recursos promovedores de comunicação e menos perturbadores, além de apresentar maior freqüência de iniciar as comunicações. Constatouse, também, que paciente e enfermeiro apresentam finalidades diferentes, e que a maior dificuldade do enfermeiro refere-se a ser responsivo ao paciente.

Díade 2: as comunicações são iniciadas, preferencialmente, pelo enfermeiro. Por outro lado, as iniciativas de comunicação efetuadas pelo paciente apontam a premência da elaboração emocional de sua condição atual, o que também pode ser confirmado pela demonstração de pouco interesse em relação às comunicações emitidas pelo profissional sobre procedimentos, dor e alimentação. Dentre as iniciativas do enfermeiro destacam-se o incentivo à participação seguida por incentivo à interação, revelando uma preocupação em favorecer uma participação mais ativa do paciente. Entretanto, nota-se, igualmente, a utilização de recursos que dificultam a expressão logo após as falas do paciente, ou seja, justamente quando este se comporta de modo mais participativo.

Díade 3: as iniciativas para comunicar têm origem nas falas do profissional e estão voltadas mais significativamente para a comunicação de informações. A emissão de recursos que dificultam é bem mais reduzida do que em outras díades. Contudo, o paciente limitou-se a apenas uma 
iniciativa de comunicação. É importante esclarecer que este atendimento foi marcado por interrupções, além do paciente ainda sentir dores.

Díade 4: esta díade foi a que menos interagiu, no decorrer do atendimento a ocorrência de pausas é superior às interações. O paciente, praticamente, não teve iniciativa de iniciar as comunicações (apenas uma ocorrência), enquanto o enfermeiro teve iniciativas voltadas para a comunicação de informações, não emitindo nenhuma iniciativa voltada para a interação. Observa-se que os recursos disponíveis tanto pelo paciente como pelo enfermeiro são escassos: os dois apresentaram as menores freqüências de recursos promovedores de comunicação e passaram a maior parte do atendimento em silêncio.

\section{COMPARAÇÃO ENTRE AS DÍADES}

As díades que apresentaram melhor índice comunicacional foram respectivamente díade 2 e díade 1 (ambas compostas pelo mesmo enfermeiro), seguidas pela díade 3 e, por último, com um índice muito mais baixo a díade 4. O melhor índice comunicacional obtido pelos enfermeiros é proveniente do enfermeiro da díade 3 , seguido pelo enfermeiro das díades 1 e 2 e por último pelo enfermeiro da díade 4. Quanto aos pacientes, o índice comunicacional obtido, em ordem decrescente, foi: P2, P1, P4 e P3. Comparando-os com os do enfermeiro, é possível verificar que não estão correlacionados positivamente.

\section{3) Percepção do enfermeiro $x$ percepção do paciente}

Enfermeiro e paciente reconhecem que a relação entre ambos é o principal elemento de satisfação com atendimento, sendo que os enfermeiros atribuem importância equivalente à organização do trabalho como fator relevante para tal satisfação. Os recursos comunicacionais são citados como fatores que podem facilitar ou dificultar a relação, por exemplo: quando o paciente é não-interativo, quando o enfermeiro não tem tempo para conversar e quando a comunicação é inadequada por falta de recursos do profissional ou por inexpressividade do paciente. Outro aspecto apontado pelo enfermeiro como vinculado a sua satisfação diz respeito a verificar que o paciente consegue perceber a existência de um objetivo comum entre eles. Vale lembrar que este dado tem sido estudado na literatura como alinhamento comunicacional (Meredith, Stewart, Brown, 2001). Chama a atenção o fato de que o resultado obtido no atendimento e a condição física do paciente foram considerados por enfermeiros e pacientes como os quesitos de menor força em sua avaliação de satisfação.

\section{4) Situação observada $x$ discurso dos participantes}

A priorização técnica é evidente tanto na situação de atendimento como no discurso profissional. Mas isso é constatável apenas quando descrevem o atendimento, pois quando comentam sobre seus objetivos, três dos enfermeiros relatam ter objetivos globais, o que revela uma contradição entre discurso e comportamento. Quanto aos pacientes, apenas dois apresentaram compatibilidade entre o observado e suas falas. Aspectos relacionados à organização do trabalho da equipe de enfermagem são mencionados nos discursos e também surgem associados às sessões de observação. Merece destaque a contradição entre o pressuposto do profissional de que o paciente deve estar bem informado e o receio de que isso acarrete uma reação depressiva, o que parece indicar a falta de preparo para lidar com a "condição psicológica do paciente". Outra contradição verificada referese ao relato dos pacientes reconhecendo que as orientações são suficientes e adequadas, mas, quando questionados sobre a existência de dúvidas, afirmam não terem tido oportunidade de esclarecê-las. Isso sugere que os pacientes superestimam as informações recebidas.

\section{CONSIDERAÇÕES FINAIS}

Verificou-se que a comunicação estabelecida entre enfermeiro e paciente cumpre três finalidades, nesta ordem de prioridade: informar, incentivar a participação e interagir. A natureza informativa, apesar de preponderante, 
não é satisfatória, havendo uma superestimação dos problemas de enfermagem e subestimação das orientações. O incentivo à participação do paciente no atendimento é realizado, pelo enfermeiro, por meio de solicitação de informações em detrimento de solicitação de atuação. A busca de interação é maior por parte do paciente e com utilização de recursos auto-referenciados que expressam seus valores e sentimentos, enquanto os enfermeiros quase não cumprem esta finalidade.

O reconhecimento da comunicação como instrumento do trabalho e a valoração dos aspectos relacionais estão mais presentes no discurso do que na prática. A utilização de recursos comunicacionais que dificultam ou favorecem o atendimento também é um indicador de que as prioridades do enfermeiro e paciente diferem. Ambos apresentam os dois tipos de recursos, mas os utilizam diferentemente. $\mathrm{O}$ enfermeiro está mais disponível para conversar sobre questões técnicas, quando a comunicação é referente a "outra finalidade", mas ao mesmo tempo ele intensifica o uso de perturbadores comunicacionais. Já o paciente ou é passivo respondendo estritamente ao que é perguntado ou busca a interação, utilizando os recursos "emitir opiniões" e "mantenedores" do contato com o interlocutor. Verificou-se que o comportamento verbal do paciente influencia o desempenho comunicacional do enfermeiro, mas não o determina. Constatou-se variação de desempenho do mesmo enfermeiro com pacientes diferentes, como também um bom desempenho de um determinado enfermeiro com um paciente que apresenta poucos recursos comunicacionais em comparação aos outros pacientes.

O tempo do atendimento é subutilizado pelo profissional, que tende a supervalorizar demandas externas que acabam por interromper o atendimento. Isso pode sugerir a existência de dificuldades para interagir com o paciente, propiciando uma disponibilidade de envolvimento com situações externas. Nesse sentido, o argumento de que falta tempo para interagir não parece ser suficiente para explicar as limitações relacionais. Pelo que foi detectado na pesquisa, "características da clientela" e "contexto do tratamento de reabilitação" contribuem de maneira mais significativa para a conduta do enfermeiro. Falta de preparo para lidar com o outro gera sentimentos de insatisfação e impotência, prejudicando o encontro terapêutico entre enfermeiro e paciente. Mais ainda, a ênfase sobre tais aspectos mostra que o profissional é bastante mobilizado emocionalmente, cobrando de si mesmo uma prática assistencial fundamentada em valores próprios do modelo de saúde integrada. Mas, sem poder contar com recursos pessoais e institucionais para fazê-lo, deposita na organização do trabalho suas expectativas de alcançar essa meta.

Do ponto de vista metodológico, é fundamental salientar a necessidade de estudos sobre a competência comunicacional que desenvolvam uma análise seqüencial, em razão justamente da discrepância evidenciada entre comportamento e discurso sobre a relação profissional-paciente. Sugere-se, ainda, uma abordagem multimetodológica com uso de observação direta e entrevista. Futuras pesquisas poderão adotar ou adaptar os sistemas de categorização propostos na presente pesquisa. $\mathrm{O}$ arcabouço metodológico construído também pode se constituir em instrumento útil para a formação profissional visando o desenvolvimento de habilidades comunicacionais em saúde.

\section{REFERÊNCIAS}

Abdo, C. H. N. (1996). Armadilhas da comunicação. São Paulo: Lemos.

Cosnier, J., Grosjean, M., \& Lacoste, M. (Org.). (1994). Soins et communication: Approches interactionistes des relations de soins. Lyon: Presses Universitaires de Lyon.

Grant, C. H., Cissna, K. N., \& Rosenfeld, L. B. (2000). Patients perceptions of physicians communication and outcomes of the accrual to trial process. Health Communication, 12(1), 23-29.

Inui,T. S., \& Carter, W. B. (1985). Problems and prospects for health services research on provider-patient communication. Medical Care, 5(23), 521-538.

Kaplan, R. (1997). Health outcomes and communication research. Health Communication, 9(1), 75-82.

Kennedy, P., Marsh, N., Lowe, R., Grey, N., Short, E., \& Rogers, B. (2000). A longitudinal analysis of psychological impact and coping strategies following spinal cord injury. British Journal of Health Psychology, 5, 157-172. 
Lambert, B. L., Street Jr., R. L., Cegala, D. J., Smith, D. H., Kurtz, S., \& Schoefield, T. (1997). Provider-patient communication, patientcentered care and the mangle of practice. Health Communication, 9(1), 27-43.

Lammers, J. C., \& Geist, P. (1997). The transformation of caring in the light and shadow of "managed care". Health Communication, 9(1), 45-60.

Makoul, G.; Arntson, P., \& Schofield, T. (1995). Health promotion in primary care: Physician patient communication and decision-making about prescription medications. Social Science and Medicine, 41(9), 1241-1254.

McNeilis, K. S. (2001). Analyzing communication competence in medical consultations. Health Communication, 13(1), 5-18.

Meredith, L., Stewart, M., \& Brown , J. B. (2001). Patient-centered communication scoring method report on nine coded interviews. Health Communication, 13(1), 19-31.

Pereira, M. E. M. S. M., \& Araujo, T. C. C. F. (2005). Estratégias de enfrentamento na reabilitação do traumatismo raquimedular. Arquivos de Neuro-Psiquiatria, 63(2-B), 502-507.

Queiroz, E. \& Araujo, T. C. C. F. (2007). Trabalho em equipe: Um estudo multimetodológico em instituição hospitalar de reabilitação. Revista Interamericana de Psicologia, 41(2), 221-230.

Ramos, V. S. C. (1998). A participação do paciente de reabilitação nas decisões, sobre seu tratamento. Dissertação de mestrado, Universidade de Brasília, Brasília.

Reilly, C. (2001). Transdisciplinary approach: An atypical strategy for proving outcomes in rehabilitative and long-term acute care settings. Rehabilitation Nursing, 26, 216-220.

Sharf, B. F., \& Street Jr., R. L. (1997). The patient as a central construct: Shifting the emphasis. Health Communication, 9(1), 1-11.
Shaikh, A., Knobloch, L. M., \& Stiles, W. B. (2001). The use of a verbal response mode coding system in determining patient and physician roles in medical interviews. Health Communication, 13(1), 49-60.

Silva, M. J. P. (1996). Comunicação tem remédio: A comunicação nas relações interpessoais de saúde. São Paulo: Gente.

Sliwa, J. A., Makoul, G., \& Betts, H. (2002). Rehabiltation-specific commnunication skills training: improving the physician-patient relationship. American Journal of Physical Medicine and Rehabilitation, 81(2), 126-132.

Street Jr., R. L., \& Millay, B. (2001). Analyzing patient participation in medical encounters. Health Communication, 13(1), 61-73.

Takman, C. A. S., \& Severinson, E. I. (1999). A description of health care professionals' experiences of encounters with patients in clinical setting. Journal of Advance Nursing, 30(6), 1368-1374.

Tran, A. N., Haidet, P., Street Jr., R .L., O’Malley, K. J., Martin, F., \& Ashton, C. M. (2004). Empowering communication: a communitybased intervention for patients. Patient Education and Counseling, 52(1), 1113-1121.

Trexler, L. E., \& Fordyce, D. J. (2000). Psychological perspectives on rehabilitation: contemporary assessment and intervention strategies. In: R. L. Braddom (Org.). Physical Medicine and Rehabilitation. (pp. 75-92). Philadelphia: Saunders Company.

Waitzkin, H. (1990). On studying the discourse of medical encounters: a critique of quantitative and qualitative methods and a proposal for reasonable compromise. Medical Care, 28(6), 473-488.

Recebido: 17/10/2007 Received: 10/17/2007

Aprovado: 03/03/2008 Approved: 03/03/2008 